\title{
Acute Gastrointestinal Bleeding in COVID-19 Patients: A Systematic Review and Meta-Analysis
}

\author{
Umair Iqbal ${ }^{1}$, Hafsa Anwar ${ }^{2}$, Hafiz Umair Siddiqui ${ }^{3}$, Muhammad Ali Khan ${ }^{4}$, Faisal Kamal ${ }^{5}$, Bradley D. Confer ${ }^{1}$ and Harshit S. Khara ${ }^{1}$ \\ ${ }^{1}$ Department of Gastroenterology and Hepatology, Geisinger Medical Center, Danville, PA, ${ }^{2}$ Department of Internal Medicine, \\ Capital Health Regional Medical Center, Trenton, NJ, ${ }^{3}$ Department of Surgery, Cleveland Clinic Foundation, Cleveland, OH, \\ ${ }^{4}$ Department of Gastroenterology and Hepatology, The University of Texas, MD Anderson Cancer Center Houston, TX, ${ }^{5}$ Department of \\ Gastroenterology and Hepatology, University of Tennessee at Memphis Health Sciences Center, Memphis, TN, USA
}

Background/Aims: More than 100 million people to date have been affected by the coronavirus disease-2019 (COVID-19) pandemic. Patients with COVID-19 have a higher risk of bleeding complications. We performed a systematic review and metaanalysis to evaluate the outcomes of COVID-19 patients with signs and symptoms of acute gastrointestinal bleeding (GIB).

Methods: A systematic literature search was carried out for articles published until until November 11, 2020, in the Embase, MEDLINE, Web of Science, and Cochrane Library databases. We included studies on COVID-19 patients with signs and symptoms of GIB.

Results: Our search yielded 49 studies, of which eight with a collective 127 patients (86 males and 41 females) met our inclusion criteria. Conservative management alone was performed in $59 \%$ of the patients, endoscopic evaluation in $31.5 \%$, and interventional radiology (IR) embolization in 11\%. Peptic ulcer disease was the most common endoscopic finding, diagnosed in $47.5 \%$ of the patients. Pooled overall mortality was $19.1 \%$ (95\% confidence interval [CI]; $12.7 \%-27.6 \%)$ and pooled mortality secondary to GIB was 3.5\% (95\% CI; $1.3 \%-9.1 \%)$. The pooled risk of rebleeding was $11.3 \%$ (95\% CI; $6.8 \%-18.4 \%$ ).

Conclusions: The majority of COVID-19 patients with GIB responded to conservative management, with a low mortality rate associated with GIB and the risk of rebleeding. Thus, we suggest limiting endoscopic and IR interventions to those with hemodynamic instability and those for whom conservative management was unsuccessful. Clin Endosc 2021;54:534-541

Key Words: Acute gastrointestinal bleeding; COVID-19; Mortality; Novel Coronavirus; Rebleeding

\section{INTRODUCTION}

The coronavirus disease-2019 (COVID-19) pandemic has affected more than 100 million individuals worldwide to date, with the highest number reported in the United States, which has more than 29 million cases. Fever, cough, and the loss of sensation of taste and smell are some of the most commonly

Received: February 5, 2021 Revised: March 5, 2021

Accepted: March 24, 2021

Correspondence: Umair Iqbal

Department of Gastroenterology and Hepatology, Geisinger Medical Center, Danville, PA 17822, USA

Tel: +570-271-6856, Fax: +570-271-6852, E-mail: umairiqbal_dmc@hotmail.com ORICD: https://orcid.org/0000-0001-5857-9391

(c) This is an Open Access article distributed under the terms of the Creative Commons Attribution Non-Commercial License (http://creativecommons.org/ licenses/by-nc/3.0) which permits unrestricted non-commercial use, distribution, and reproduction in any medium, provided the original work is properly cited. presenting symptoms. ${ }^{1}$ However, studies have shown that a large number of patients present with gastrointestinal symptoms, such as diarrhea, nausea, and abdominal pain. ${ }^{2,3}$ Acute gastrointestinal bleeding (GIB) is one of the most common causes of gastrointestinal consultation in hospitalized patients in the United States. ${ }^{4}$ Patients with COVID-19 are at a higher risk of thrombotic and bleeding complications due to various reasons, such as the use of anticoagulants for the prevention and treatment of thrombotic complications, the use of corticosteroids, and mechanical ventilation. ${ }^{5,6}$

Due to the high transmissibility of this novel coronavirus and shortage of personal protective equipment (PPE), there has been an increase in anxiety among healthcare workers regarding the treatment of these patients. ${ }^{7.8}$ Endoscopic evaluation is usually recommended in patients with suspected GIB within $24 \mathrm{~h}$ of presentation. ${ }^{9,10}$ However, in patients with COVID-19, the decision to perform endoscopy has been chal- 
lenging due to the high risk of transmission; therefore, some clinicians pursue conservative management. Limited data are available regarding the outcomes of GIB in these patients, and the evidence is limited to small observational studies, case series, and case reports. ${ }^{11-21}$ Therefore, we performed a systematic review and meta-analysis to evaluate the etiology, management, and outcomes of COVID-19 patients who had signs and symptoms of GIB.

\section{MATERIALS AND METHODS}

A systematic literature search was performed from December 2019 to November 11, 2020, in the Embase, MEDLINE, Web of Science, and Cochrane Library databases. Details of search terms utilized for systematic searches in each database are listed in the Supplementary File 1. Two authors (Umair Iqbal and Harshit S. Khara) independently conducted a systematic search. All studies that included five or more COVID-19 patients with signs and symptoms of GIB were included. The included studies were observational studies. We excluded individual case reports and case series with fewer than five patients. We also excluded articles that did not report the management and outcomes of COVID-19 patients with GIB. There were no language restrictions for the study to be included in the meta-analysis.

From the included studies, we extracted the author's name, study year and country, age and gender of the included patient population, total sample size, medical comorbidities, signs and symptoms of GIB, if endoscopic evaluation was performed, if patients required endoscopic intervention, endoscopic findings, management of bleeding, and mortality. Our primary outcomes of interest were overall mortality in COVID-19 patients with GIB and mortality secondary to GIB. The secondary outcome was rebleeding rate.

We performed a quality assessment of the individual study using the New Castle Ottawa Quality assessment score to evaluate the quality of cohort studies and National Institutes of Health quality assessment tools to evaluate the quality of the case series. Two authors independently evaluated the quality of the study. The quality of the study did not interfere with its inclusion in the meta-analysis. This meta-analysis was performed according to the Preferred Reporting Items for Systematic Reviews and Meta-Analyses (PRISMA) guidelines. ${ }^{22}$ The PRISMA checklist is provided in the Supplementary File 2.

\section{Statistical analysis}

Statistical analysis was conducted using Comprehensive Meta-Analysis software (Biostat version 3, Englewood, NJ, USA).
A random-effects model was utilized for this meta-analysis, with point estimates, variance, and weights for each study based on the size of the study and the number of events. Weighted pooled rates along with $95 \%$ confidence intervals (CI) were calculated for the primary outcomes of interest, which were overall mortality and mortality secondary to GIB. Statistical significance was set at $p<0.05$. The heterogeneity of the studies was evaluated using the $I^{2}$ test.

\section{RESULTS}

An initial search yielded 49 studies. After duplicates were removed, 29 studies underwent title and abstract review, of which 24 studies were selected for full-text review. Out of those eight studies, 127 COVID-19 patients met our inclusion criteria. ${ }^{20,21,23-28}$ Fig. 1 elaborates on the systematic search process of our meta-analysis. Six of the included studies were of good quality, and two were of fair quality. There were 86 males in this study. Table 1 reports the baseline characteristics, including age, Glasgow Blatchford score, and chronic comorbidities of the included patients.

From the reported data, $80 \%$ of the patients were on either prophylactic or therapeutic anticoagulants, and 76.3\% (97/127) had confirmed or suspected upper GIB (UGIB) while 23.7\% (30/127) had confirmed or suspected lower GIB (LGIB). Conservative management alone, without endoscopic or interventional radiology evaluation, was performed in 59\% (75/127) of the patients. Endoscopic evaluation was performed in 31.5\% (40/127); specifically, 35 with esophagogastroduodenoscopy (EGD), 4 with sigmoidoscopy, and 1 with colonoscopy. Peptic ulcer disease (PUD) was the most common endoscopic finding (47.5\% of the patients), followed by gastritis (10\%) and esophagitis (7.5\%). Rectal ulcers secondary to the rectal tubes were the most frequent finding in patients who underwent lower endoscopic evaluation (three out of five patients). All three patients were managed with rectal packing via colorectal surgery.

Endoscopic intervention was performed in $32.5 \%(13 / 40)$ of patients who underwent endoscopy, of whom $85 \%$ (11/13) required intervention for PUD, one required argon plasma coagulation (APC) for gastric antral vascular ectasia (GAVE), and one required cyanoacrylate injection for isolated gastric varices type 1 . Interventional radiology-guided angioembolization was performed in $11 \%(14 / 127)$ of the patients, of whom $86 \%(12 / 14)$ were hemodynamically unstable and $14 \%$ $(2 / 14)$ had rebleeding. Table 1 reports a detailed description of the endoscopic findings and interventions performed in the COVID-19 patients. 
C clinical endoscopy

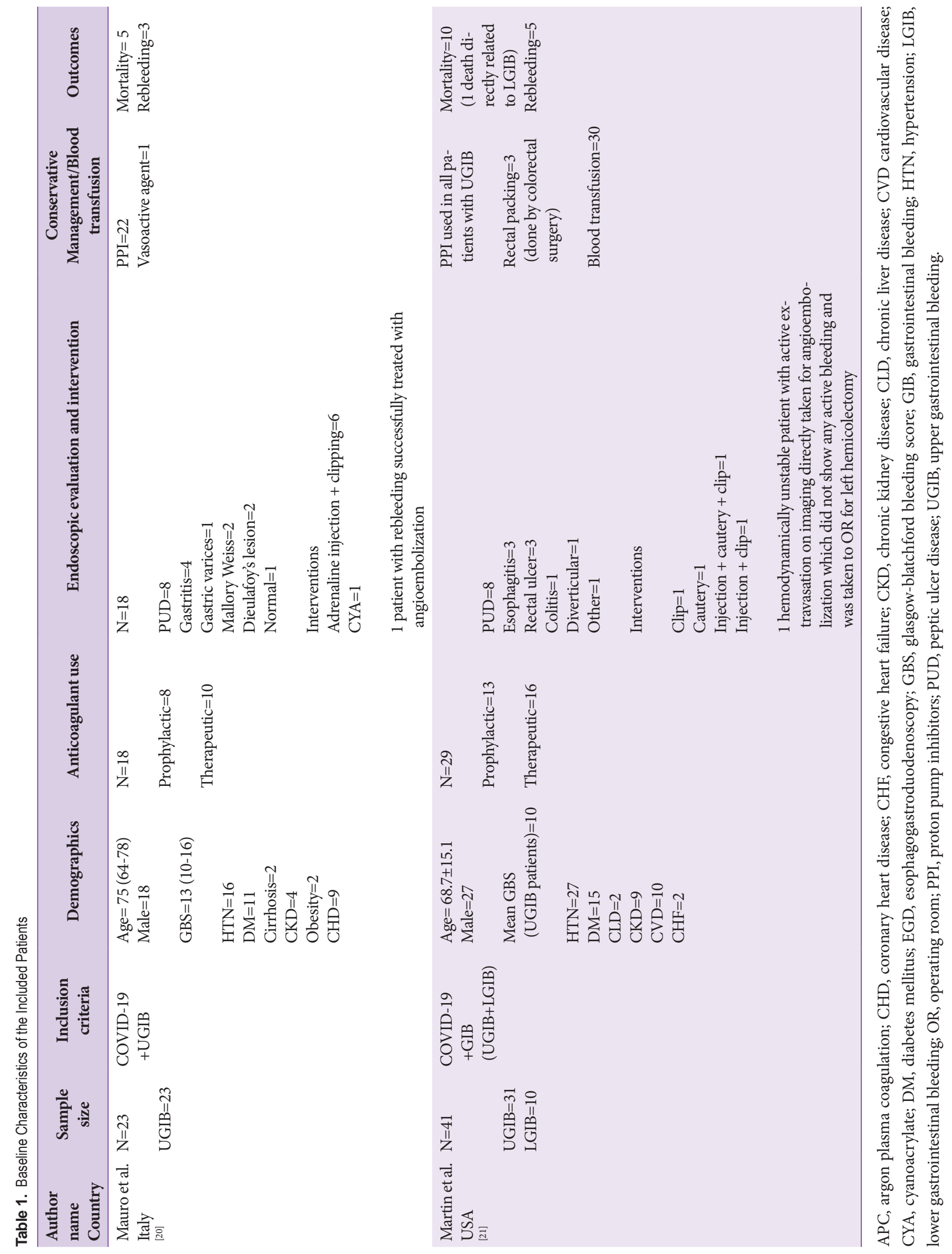




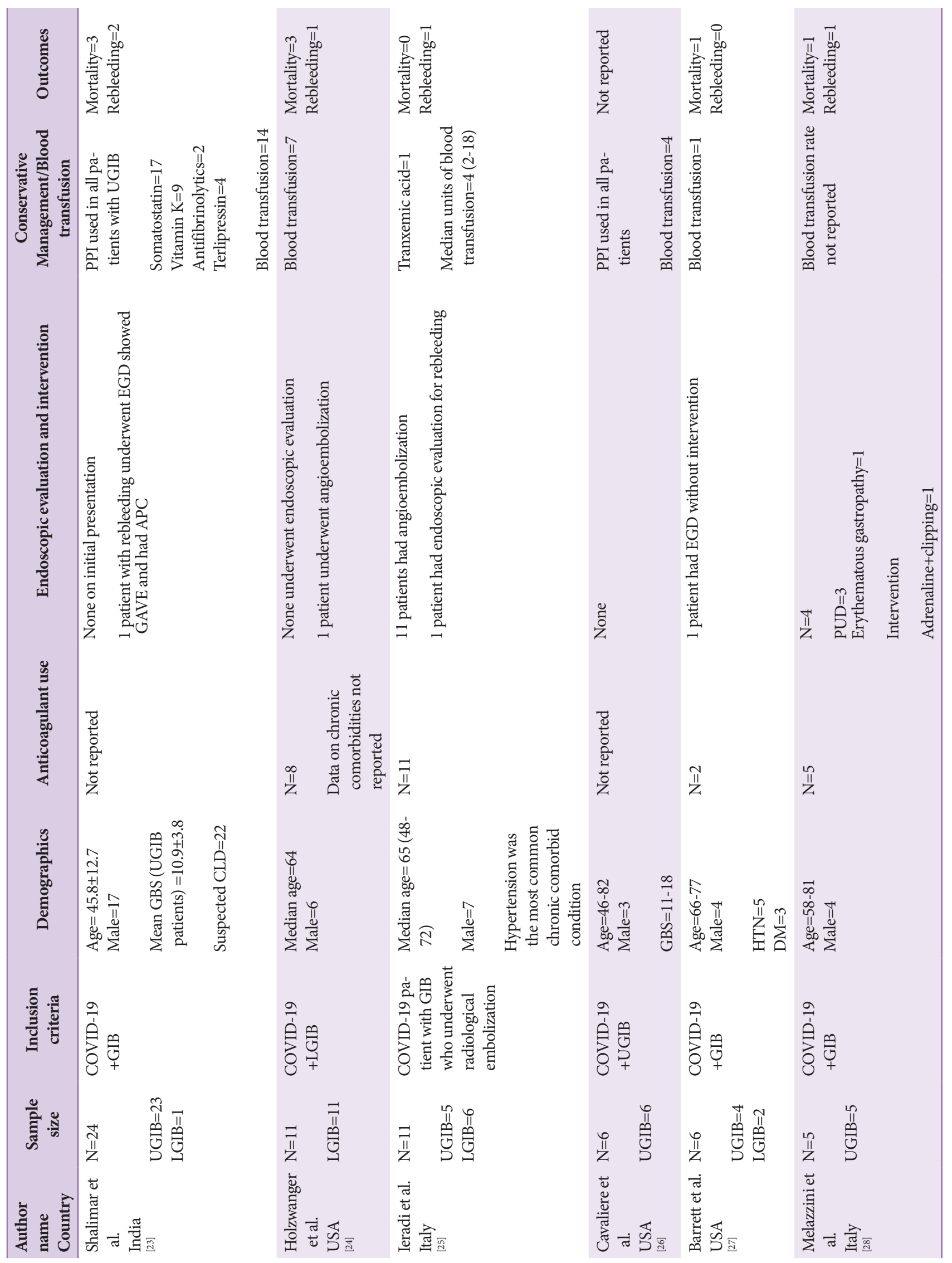



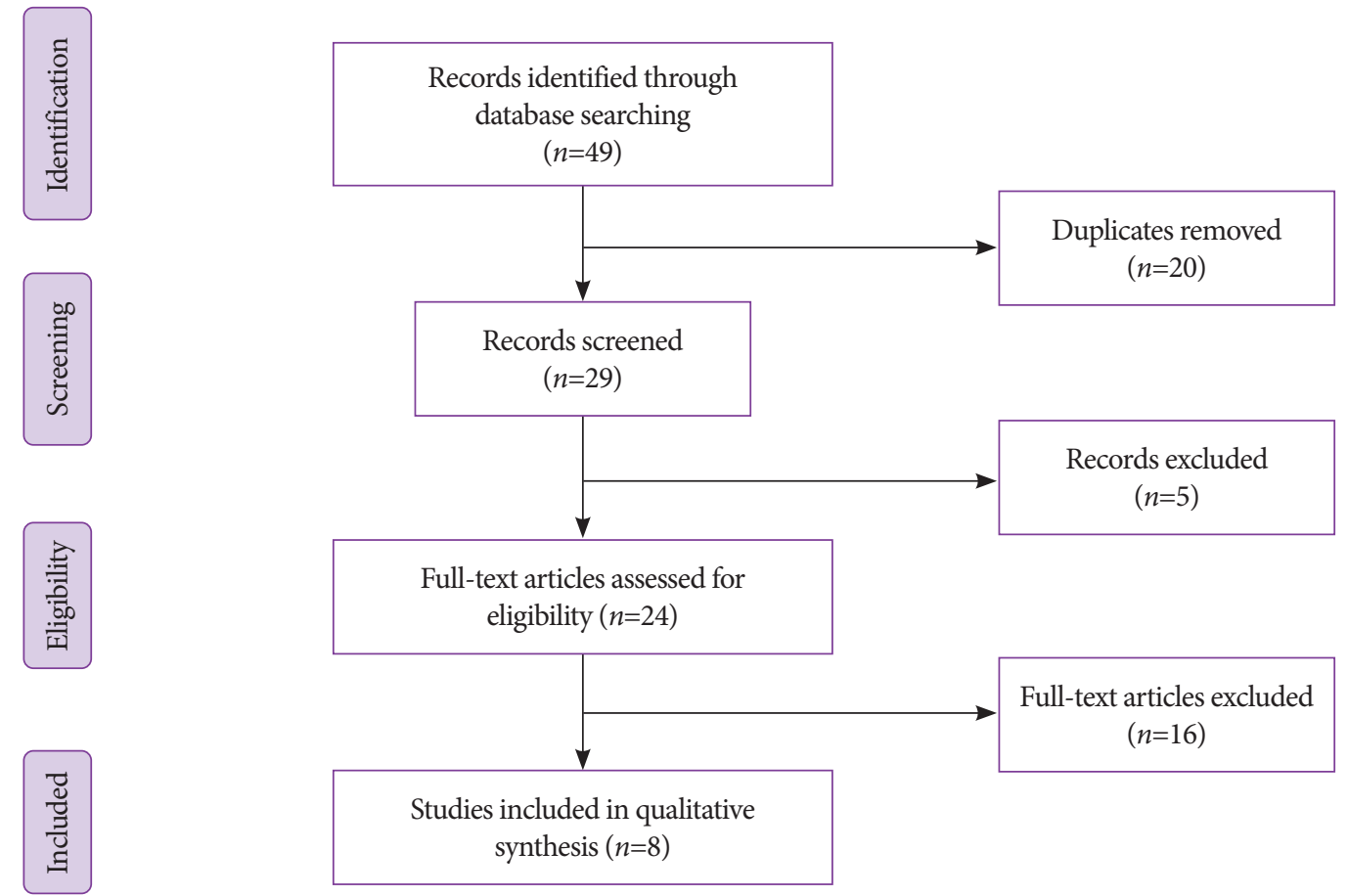

Fig. 1. Literature review process.

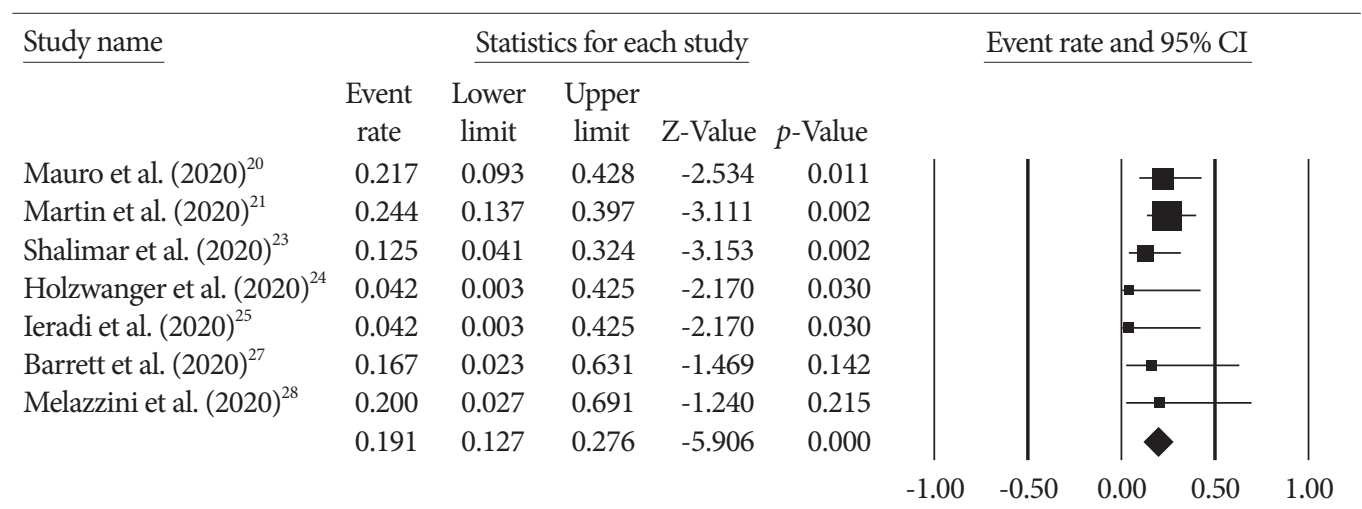

Fig. 2. Forest plot for overall mortality. $\mathrm{Cl}$, confidence interval.

The pooled overall mortality was $19.1 \%$ (95\% CI; $12.7 \%$ 27.6\%) with $I^{2}=0$ (Fig. 2). The majority of the patients died secondary to COVID-19 complications. Only one patient died secondary to GIB and hemorrhagic shock. Pooled mortality secondary to GIB was 3.5\% (95\% CI; 1.3\%-9.1\%) (Fig. 3). The pooled risk of rebleeding was $11.3 \%$ (95\% CI; 6.8\%-18.4\%) with no heterogeneity (Fig. 4). 


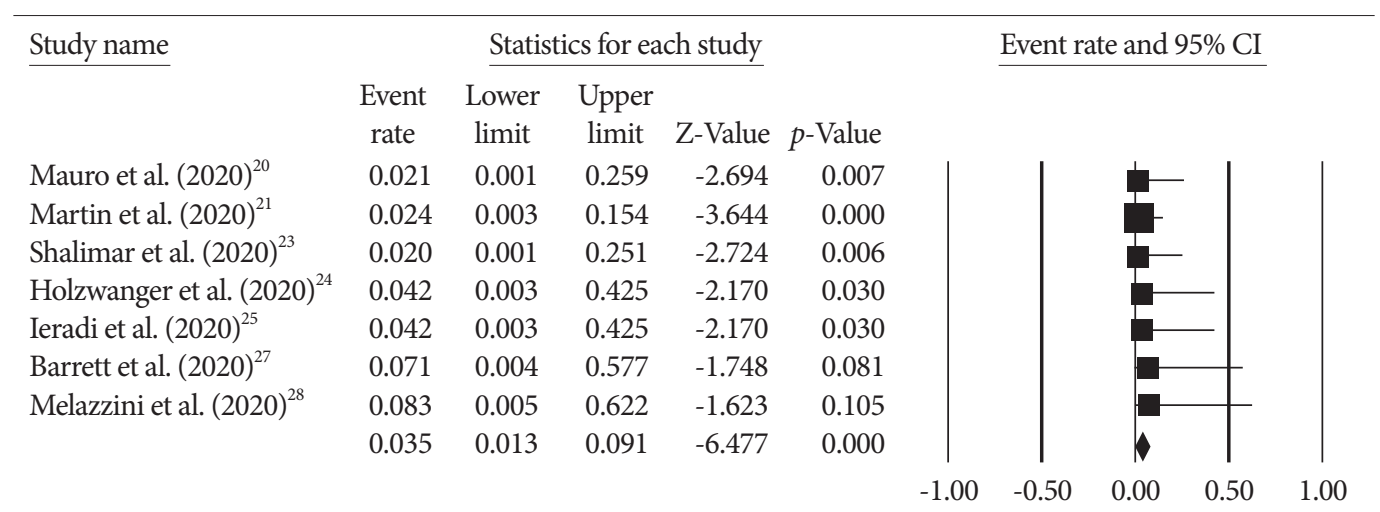

Fig. 3. Forest plot for gastrointestinal bleeding-related mortality. $\mathrm{Cl}$, confidence interval.

\begin{tabular}{|c|c|c|c|c|c|c|c|c|}
\hline \multirow[t]{2}{*}{ Study name } & \multicolumn{5}{|c|}{ Statistics for each study } & & \multicolumn{2}{|c|}{ Event rate and $95 \% \mathrm{CI}$} \\
\hline & $\begin{array}{c}\text { Event } \\
\text { rate }\end{array}$ & $\begin{array}{c}\text { Lower } \\
\text { limit }\end{array}$ & $\begin{array}{c}\text { Upper } \\
\text { limit }\end{array}$ & Z-Value & $p$-Value & & & \\
\hline Mauro et al. $(2020)^{20}$ & 0.130 & 0.043 & 0.335 & -3.064 & 0.002 & & & \\
\hline Martin et al. $(2020)^{21}$ & 0.122 & 0.052 & 0.261 & -4.136 & 0.000 & & & \\
\hline Shalimar et al. $(2020)^{23}$ & 0.083 & 0.021 & 0.279 & -3.247 & 0.001 & & & $\bar{a}$ \\
\hline Holzwanger et al. $(2020)^{24}$ & 0.091 & 0.013 & 0.439 & -2.195 & 0.028 & & & 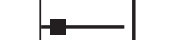 \\
\hline Ieradi et al. $(2020)^{25}$ & 0.091 & 0.013 & 0.439 & -2.195 & 0.028 & & & \\
\hline Barrett et al. $(2020)^{27}$ & 0.071 & 0.004 & 0.577 & -1.748 & 0.081 & & & \\
\hline \multirow[t]{3}{*}{ Melazzini et al. $(2020)^{28}$} & 0.200 & 0.027 & 0.691 & -1.240 & 0.215 & & & $\rightarrow$ \\
\hline & 0.113 & 0.068 & 0.184 & -7.097 & 0.000 & & & 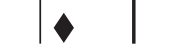 \\
\hline & & & & & & -1.00 & -0.50 & 0.00 \\
\hline
\end{tabular}

Fig. 4. Forest plot for rebleeding. $\mathrm{Cl}$, confidence interval.

\section{DISCUSSION}

We performed this meta-analysis to delineate the current literature at the time of writing on COVID-19 patients with GIB. Our meta-analysis aimed to help clinicians, especially gastroenterologists, in their decision-making for the management of GIB in COVID-19 patients. These patients have a high prevalence of GI symptoms and the involvement of the gastroenterologist in their management is highly likely. ${ }^{2,3,29}$ A study conducted on 95 patients with COVID-19 showed the presence of GI symptoms in $61 \%$ of the participants, with diarrhea, nausea, and anorexia being the most common symptoms. ${ }^{29}$ There is evidence of good quality that supports the association of COVID-19 with increased risk of thrombotic complications. The use of anticoagulants is more common in these patients. ${ }^{6,30}$ In addition, steroids have been shown to be beneficial in the treatment of COVID-19 patients. ${ }^{5,31}$ Critically ill patients with COVID-19 also require mechanical ventilation and are at higher risk of stress ulcers. ${ }^{32,33}$ All of these factors in combination increase patients' risk of GIB.
Our meta-analysis revealed that the majority of patients responded to conservative management with intravenous hydration, blood transfusion, proton pump inhibitor use in UGIB, and rectal packing in patients suspected of having rectal ulcers. For patients who required endoscopic evaluation, only onethird required endoscopic intervention. The overall mortality was high but there was only one death directly related to GIB, whereas mostly were secondary to COVID-19 complications. ${ }^{21}$ In a study of 24 patients with GIB and COVID-19 (23 UGIB, 1 LGIB, and 22 suspected chronic liver disease), conservative management without endoscopic evaluation resulted in the resolution of GIB in all patients. ${ }^{23}$ No mortality was reported and rebleeding was reported in only two patients. One of them required EGD on day 18 of initial presentation and was found to have GAVE, which was successfully treated with APC. ${ }^{23}$ In another study of 11 COVID-19 patients with LGIB, all except one were treated with conservative management without any rebleeding episodes. ${ }^{24}$ Only one hemodynamically unstable patient required angioembolization. A study of 38 patients who underwent 44 endoscopic evaluations (24 EGDs and 
20 colonoscopies) for various indications revealed PUD and esophagitis as the common finding on EGD, which was consistent with our study results. Colitis and colonic ischemia were the most frequent findings on colonoscopy examination. $^{34}$ It is important to note that none of the endoscopists in this study were infected post-procedure with the utilization of PPE, including a filtering face piece, goggles, two pairs of gloves, and surgical gowns. Most patients required endoscopic evaluation for the indication of GIB. This study did not report the number of patients who underwent endoscopy for the indication of GIB, and the number of patients who needed endoscopic intervention; therefore, we did not include this study in our meta-analysis. ${ }^{34}$

The systematic literature search of our study is comprehensive and will provide concise information regarding the available literature to date on the management of GIB in COVID-19 patients. However, there are some limitations to our study, and the results should be interpreted with caution. The sample size of most of the included studies was small, and all of the included studies were retrospective observational studies, which may introduce bias to the results. Therefore, there is a need for a prospective study with a large sample size to further evaluate the outcomes of GIB in COVID-19 patients. Due to a lack of reporting of data, we were unable to meta-analytically evaluate differences in mortality and rate of blood transfusion between patients who underwent endoscopic evaluation or angioembolization and those who were managed conservatively. In a multicenter study comparing 41 COVID-19 patients with GIB and 82 matched COVID-19 controls without GIB, no statistically significant difference was observed between the respective in-hospital mortalities. There was also no statistically significant difference in blood transfusion requirement in UGIB between those who underwent endoscopic evaluation or intervention and in those who were managed conservatively. ${ }^{21}$ However, we found conservative management to be effective in most of the patients with only one death secondary to GIB. In addition, there might be decreased reporting of cases with negative outcomes with conservative management; thus, mortality directly secondary to GIB may actually be higher. Last, information on COVID-19 is a rapidly changing topic, and the publication of further data may change our findings in the future.

\section{CONCLUSIONS}

The majority of COVID-19 patients with GIB were managed conservatively. PUD was the most common etiology, and endoscopic intervention was required in only one-third of the patients who underwent endoscopic evaluation. The overall mortality rate was high, but only one death was directly related to GIB. We suggest considering conservative management in hemodynamically stable COVID-19 patients who have GIB with PPI, blood transfusion, and intravenous hydration. We also suggest considering endoscopic evaluation in hemodynamically unstable patients in whom conservative management was unsuccessful, and hemodynamically stable patients at risk of hemodynamic instability, depending on the clinical assessment of the patient. Prospective studies are needed to further evaluate the management and outcomes of GIB in patients with COVID-19.

Conflicts of Interest

The authors have no potential conflicts of interest.

Funding

None.

Acknowledgments

We would like to thank Matthew Roslund at Bassett Medical Center for validation of the search.

ORCID
Umair Iqbal:

Hafsa Anwar:

Hafiz Umair Siddiqui:

Muhammad Ali Khan:

Faisal Kamal:

Bradley D. Confer:

Harshit S Khara: https://orcid.org/0000-0001-5857-9391 https://orcid.org/0000-0003-0427-9128 https://orcid.org/0000-0002-7542-5084 https://orcid.org/0000-0001-8235-1733 https://orcid.org/0000-0002-6842-506X https://orcid.org/0000-0002-1409-3979 https://orcid.org/0000-0003-2850-625X

\section{REFERENCES}

1. Grant MC, Geoghegan L, Arbyn M, et al. The prevalence of symptoms in 24,410 adults infected by the novel coronavirus (SARS-CoV-2; COVID-19): a systematic review and meta-analysis of 148 studies from 9 countries. PLoS One 2020;15:e0234765.

2. Suresh Kumar VC, Mukherjee S, Harne PS, et al. Novelty in the gut: a systematic review and meta-analysis of the gastrointestinal manifestations of COVID-19. BMJ Open Gastroenterol 2020;7:e000417.

3. Buscarini E, Manfredi G, Brambilla G, et al. GI symptoms as early signs of COVID-19 in hospitalised Italian patients. Gut 2020;69:1547-1548.

4. Peery AF, Crockett SD, Murphy CC, et al. Burden and cost of gastrointestinal, liver, and pancreatic diseases in the United States: update 2018. Gastroenterology 2019;156:254-272.e11.

5. WHO Rapid Evidence Appraisal for COVID-19 Therapies (REACT) Working Group, Sterne JAC, Murthy S, et al. Association between administration of systemic corticosteroids and mortality among critically ill patients with COVID-19: a meta-analysis. JAMA 2020;324:13301341.

6. Bilaloglu S, Aphinyanaphongs Y, Jones S, Iturrate E, Hochman J, Berger JS. Thrombosis in hospitalized patients with COVID-19 in a New York City Health System. JAMA 2020;324:799-801.

7. Pappa S, Ntella V, Giannakas T, Giannakoulis VG, Papoutsi E, Katsaounou P. Prevalence of depression, anxiety, and insomnia among healthcare workers during the COVID-19 pandemic: a systematic review and meta-analysis. Brain Behav Immun 2020;88:901-907. 
8. Gondi S, Beckman AL, Deveau N, et al. Personal protective equipment needs in the USA during the COVID-19 pandemic. Lancet 2020;395:e90-e91.

9. Laine L, Jensen DM. Management of patients with ulcer bleeding. Am J Gastroenterol 2012;107:345-360:quiz 361

10. Strate LL, Gralnek IM. ACG clinical guideline: management of patients with acute lower gastrointestinal bleeding. Am J Gastroenterol 2016;111:459-474.

11. Buckholz A, Kaplan A, Jessurun J, De Jong Y, Crawford C. Microthrombosis associated with GI bleeding in COVID-19. Gastrointest Endosc 2021;93:263-264.

12. Carvalho A, Alqusairi R, Adams A, et al. SARS-CoV-2 gastrointestinal infection causing hemorrhagic colitis: implications for detection and transmission of COVID-19 disease. Am J Gastroenterol 2020;115:942946.

13. Cho M, Liu W, Balzora S, et al. Clinical and intestinal histopathological findings in SARS-CoV-2/COVID-19 patients with hematochezia. Case Rep Gastroenterol 2021;15:408-417.

14. Gadiparthi C, Perisetti A, Sayana H, Tharian B, Inamdar S, Korman A. Gastrointestinal bleeding in patients with severe SARS-CoV-2. Am J Gastroenterol 2020;115:1283-1285.

15. Gulen M, Satar S. Uncommon presentation of COVID-19: gastrointestinal bleeding. Clin Res Hepatol Gastroenterol 2020;44:e72-e76.

16. Guotao L, Xingpeng Z, Zhihui D, Huirui W. SARS-CoV-2 infection presenting with hematochezia. Med Mal Infect 2020;50:293-296.

17. Li X, Huang S, Lu J, et al. Upper gastrointestinal bleeding caused by SARS-CoV-2 infection. Am J Gastroenterol 2020;115:1541-1542.

18. Marchi G, Vianello A, Crisafulli E, et al. Cytomegalovirus-induced gastrointestinal bleeding and pancreatitis complicating severe COVID-19 pneumonia: a paradigmatic case. Mediterr J Hematol Infect Dis 2020;12:e2020060

19. Zhang L-B, Pang R-R, Qiao Q-H, et al. Successful recovery of COVID-19associated recurrent diarrhea and gastrointestinal hemorrhage using convalescent plasma. Mil Med Res 2020;7:45.

20. Mauro A, De Grazia F, Lenti MV, et al. Upper gastrointestinal bleeding in COVID-19 inpatients: incidence and management in a multicenter experience from Northern Italy. Clin Res Hepatol Gastroenterol 2020;101521.

21. Martin TA, Wan DW, Hajifathalian K, et al. Gastrointestinal bleeding in patients with coronavirus disease 2019: a matched case-control study. Am J Gastroenterol 2020;115:1609-1616.
22. Moher D, Liberati A, Tetzlaff J, Altman DG, PRISMA Group. Preferred reporting items for systematic reviews and meta-analyses: the PRISMA statement. PLoS Med 2009;6:e1000097.

23. Shalimar null, Vaishnav M, Elhence A, et al. Outcome of conservative therapy in coronavirus disease-2019 patients presenting with gastrointestinal bleeding. J Clin Exp Hepatol 2021;11:327-333.

24. Holzwanger EA, Bilal M, Stallwood CG, Sterling MJ, Yacavone RF. Acute lower gastrointestinal bleeding during the COVID-19 pandemic - less is more! Endoscopy 2020;52:816-817.

25. Ierardi AM, Del Giudice C, Coppola A, et al. Gastrointestinal hemorrhages in patients with COVID-19 managed with transarterial embolization. Am J Gastroenterol 2021;116:838-840.

26. Cavaliere K, Levine C, Wander P, Sejpal DV, Trindade AJ. Management of upper GI bleeding in patients with COVID-19 pneumonia. Gastrointest Endosc 2020;92:454-455.

27. Barrett LF, Lo KB, Stanek SR, Walter JW. Self-limited gastrointestinal bleeding in COVID-19. Clin Res Hepatol Gastroenterol 2020;44:e77-e80.

28. Melazzini F, Lenti MV, Mauro A, De Grazia F, Di Sabatino A. Peptic ulcer disease as a common cause of bleeding in patients with coronavirus disease 2019. Am J Gastroenterol 2020;115:1139-1140.

29. Lin L, Jiang X, Zhang Z, et al. Gastrointestinal symptoms of 95 cases with SARS-CoV-2 infection. Gut 2020;69:997-1001.

30. Klok FA, Kruip MJHA, van der Meer NJM, et al. Incidence of thrombotic complications in critically ill ICU patients with COVID-19. Thromb Res 2020;191:145-147.

31. RECOVERY Collaborative Group, Horby P, Lim WS, et al. Dexamethasone in hospitalized patients with COVID-19. N Engl J Med 2021;384:693704 .

32. Plummer MP, Blaser AR, Deane AM. Stress ulceration: prevalence, pathology and association with adverse outcomes. Crit Care 2014;18:213.

33. PEPTIC Investigators for the Australian and New Zealand Intensive Care Society Clinical Trials Group, Alberta Health Services Critical Care Strategic Clinical Network, the Irish Critical Care Trials Group, et al. Effect of stress ulcer prophylaxis with proton pump inhibitors vs histamine-2 receptor blockers on in-hospital mortality among ICU patients receiving invasive mechanical ventilation: the PEPTIC randomized clinical trial. JAMA 2020;323:616-626.

34. Massironi S, Viganò C, Dioscoridi L, et al. Endoscopic findings in patients infected with 2019 novel coronavirus in lombardy, Italy. Clin Gastroenterol Hepatol 2020;18:2375-2377. 